\title{
DYNAMICAL MODELING OF LASER ABLATION PROCESSES
}

J. N. Leboeuf*, K. R. Chen*, J. M. Donato ${ }^{\dagger}$, D. B. Geohegan $¥$, C. L. Liu ${ }^{\ddagger}$, A. A. Puretzky

${ }^{*}$ Fusion Energy Division

†Computer Science and Mathematics Division

¥Solid State Division

Oak Ridge National Laboratory, Oak Ridge, TN 37831-8071

\section{ABSTRACT}

Several physics and computational approaches have been developed to globally characterize phenomena important for film growth by pulsed laser deposition of materials. These include thermal models of laser-solid target interactions that initiate the vapor plume; plume ionization and heating through laser absorption beyond local thermodynamic equilibrium mechanisms; gas dynamic, hydrodynamic, and collisional descriptions of plume transport; and molecular dynamics models of the interaction of plume particles with the deposition substrate. The complexity of the phenomena involved in the laser ablation process is matched by the diversity of the modeling task, which combines materials science, atomic physics, and plasma physics.

\section{INTRODUCTION}

The laser ablation technique for pulsed laser deposition of thin films is reported to be conceptually and experimentally simple [1] and yet has proven extremely successful at growing high-quality films of very complex materials, such as high-temperature superconducting compounds. The physics ingredients that come into play are also quite complicated given that they involve laser-solid interactions at the target, plasma formation off the target, vapor/plasma plume transport toward the deposition substrate with its associated hydrodynamics and atomic physics, as well as plume-solid interactions at the deposition substrate.

We have taken a global physics and computational approach to the laser ablation process that relies on thermal models to describe laser-solid interactions; on kinetic models of plasma formation in the plume; on an assorted variety of hydrodynamic, gas dynamic, and collisional, models of plume transport; as well as on molecular dynamics methods to treat plume-substrate interactions. We have chosen to concentrate mostly on silicon to validate our models. The application of our physics results does however go beyond silicon, given the universality of many experimental observations, such as plume splitting for instance [2], for a wide variety of laser-ablated materials, be it carbon, copper, yttrium or YBCO. For laser-target interactions, the issues that we have addressed with our thermal models cover the vaporization rate as a function of laser fluence and the effect of the pressure at the liquid surface on the vaporization threshold. For plasma formation, the likelihood of vapor breakdown during the laser pulse has been tackled with nonequilibrium, kinetic rate equations models. As far as plume transport is concerned, the differing character of plume dynamics in near-vacuum and in the presence of a higher pressure background gas [3] has been treated with our gas dynamic and collisional models. In the area of plume-substrate interactions, the possibility of film damage by highly energetic plume particles has been investigated with the embedded atom method of molecular dynamics.

\section{LASER-TARGET INTERACTIONS}

Thermal models of laser-solid interactions have been successfully applied to laser annealing of semiconductors [4]. As implemented in the one- and two-dimensional (1- and 2-D) Laser8 computer programs [5], these models solve the enthalpy $(\rho h=\rho e+P$, with $\rho$ the density, $h$ the enthalpy, $\mathrm{e}$ the internal energy, and $\mathrm{P}$ the pressure) diffusion equation using finite differences

$$
\begin{aligned}
& \frac{\partial}{\partial t}(\rho h)=\frac{\partial}{\partial x}\left(\kappa \frac{\partial}{\partial x} T\right)+S . \quad \text { VI }
\end{aligned}
$$

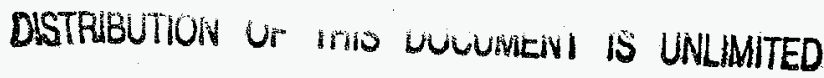

$$
\begin{aligned}
& \text { at }
\end{aligned}
$$

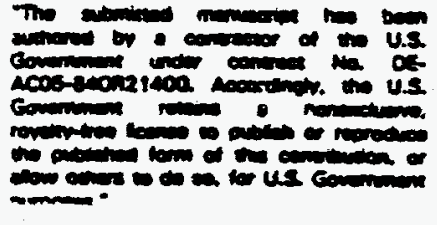




\section{DISCLAIMER}

Portions of this document may be illegible in electronic image products. Images are produced from the best available original document. 
The laser energy input is specified at each instant of time and each point in space through the source term $S$ related to the intensity of the laser pulse $\Phi$ :

$$
\mathrm{S}(\mathrm{x}, \mathrm{t})=[1-\mathrm{R}(\mathrm{x}, \mathrm{t})] \Phi(\mathrm{t}) \alpha \mathrm{e}^{-\alpha \mathrm{x}} \text {. }
$$

For silicon, the absorption coefficient $\alpha$ is taken to be $\alpha=1 \times 10^{6} \mathrm{~cm}^{-1}$ in both the solid and liquid phases; the reflectivity $\mathrm{R}$ is set at 0.58 for the solid phase and 0.69 for the liquid phase; and the profile of the thermal conductivity $K$ is as in Ref. 5. Phase transitions, which may be timedelayed, are handled through the state array concept that determines the state of each cell in space and time according to the appropriate (enthalpy $h$, temperature $T$ ) state diagram. In addition to the solid-liquid transition appropriate for laser annealing, we have extended the process to include the liquid-vapor transition necessary to model the initial stages of laser ablation. The extended thermal model also includes the effect of the pressure at the liquid surface $P_{S}$ on the vaporization temperature through the Clausius-Clapeyron equation:

$$
T_{v}=\left[\frac{1}{T_{0}}-\frac{\ln \left(P_{s} / P_{0}\right)}{\Delta H}\right]^{-1},
$$

where $T_{0}$ is the vaporization temperature at atmospheric pressure $P_{0}$ and $\Delta H$ is the latent heat.

Results from calculations with the 1-D version of the Laser8 computer program are displayed in Fig. 1. The depth of vaporization and maximum recession speed at atmospheric pressure as a function of laser energy density are displayed on the right. Both are linear with energy density and indicate that the vaporization threshold is around $4 \mathrm{~J} / \mathrm{cm}^{2}$. The vaporization temperature and threshold laser energy density for onset of vaporization as a function of pressure at the liquid surface $P_{S}$ are displayed on the left. Both go like $\log \left(P_{S}\right)$ and indicate that a lower vaporization temperature and a lower threshold laser energy density are obtained in near-vacuum than at atmospheric pressure in accordance with the Clausius-Clapeyron equation. In particular, the predicted threshold laser energy density decreases from $\sim 4 \mathrm{~J} / \mathrm{cm}^{2}$ at atmospheric pressure to $\sim 1.5 \mathrm{~J} / \mathrm{cm}^{2}$ at a pressure of $1 \mathrm{mT}$ Torr.
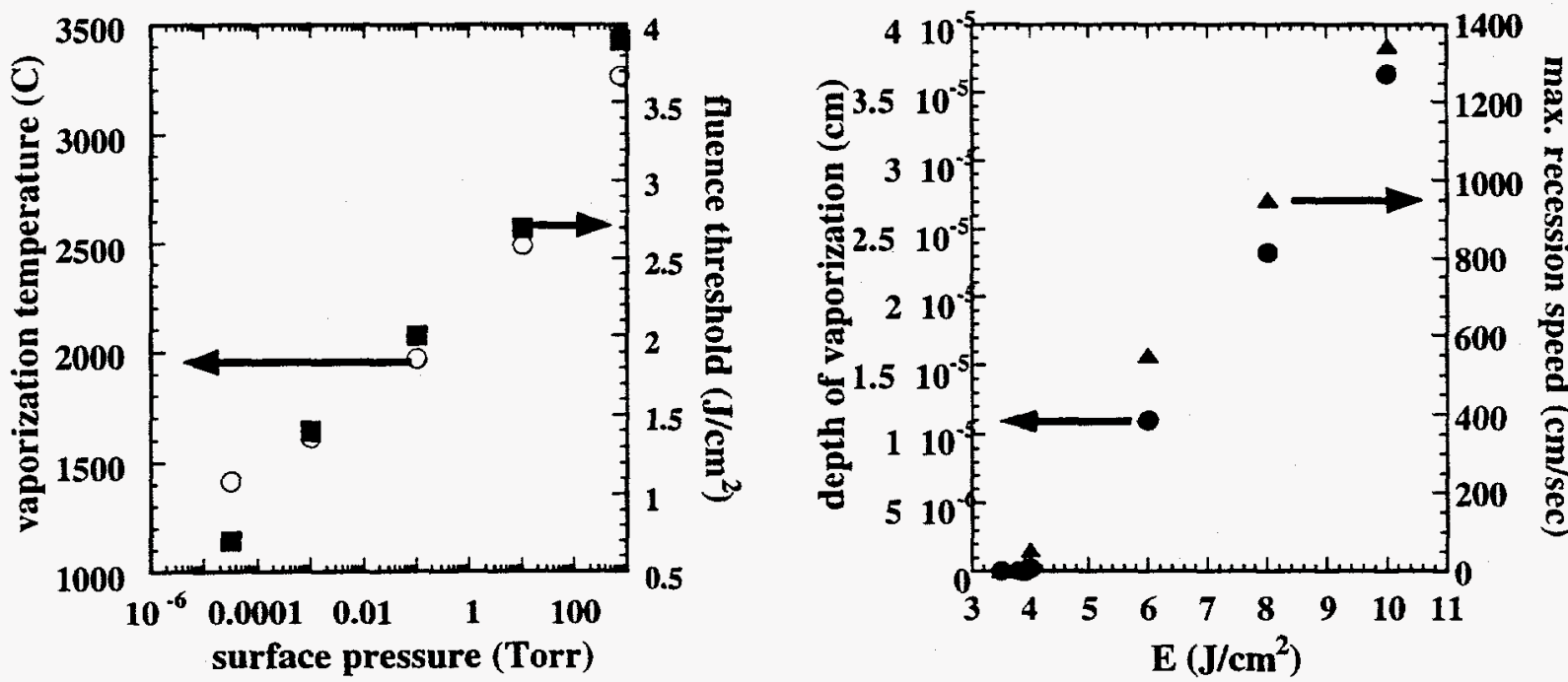

Fig. 1 Results from 1-D Laser8 for silicon: vaporization temperature and laser energy density threshold for vaporization as a function of surface pressure (left) and vaporization depth and maximum recession speed as a function of laser fluence at a liquid surface pressure of 1 atm (right). 
The thermal model allows us to specify the initial conditions for the plume transport models to be described next.

\section{PLUME FORMATION AND TRANSPORT}

\section{Plasma formation}

Before considering gas dynamic and collisional models of plume transport from the target to the deposition substrate, let us briefly discuss the issue of plasma formation. From local thermodynamic equilibrium considerations, it has proven difficult to ionize the silicon vapor plume at the typical vaporization temperature of $0.3 \mathrm{eV}$. Even for a neutral density as low as $1015 \mathrm{~cm}^{-3}$, an ionization fraction of only 0.001 is obtained according to the Saha equation. The lack of electrons makes absorption of laser light by the vapor unlikely. According to the best formulas available for electron-neutral inverse Bremstrahlung [6], electron-ion inverse Bremstrahlung [6], and plasma resonance absorption [7], the first is the largest of the three but at least 9 orders of magnitude smaller than the absorption coefficient in the solid and liquid phase for the most optimistic ionization fraction at the nominal vaporization temperature of $0.3 \mathrm{eV}$. The last is not important for $\mathrm{KrF}$ lasers since the critical plasma density required is close to solid density and therefore much greater than the expected plume densities at the end of the laser pulse.

These considerations have prompted us to investigate nonequilibrium processes for plume ionization and absorption of laser light by the plume. Our breakdown model for 248-nm KrF laser light interacting with silicon within a pulse length of $\sim 40 \mathrm{~ns}$ is described in detail in a companion paper [8] and will only be briefly reviewed here. It is based on a kinetic model of breakdown first applied by Rosen et al. [9] to coupling of $1-\mu$ s pulses of $0.35-\mu \mathrm{m}$ XeF laser radiation to aluminum alloys. Rate equations for densities and temperatures of electrons, excited state neutrals, ground state neutrals and singly charged silicon ions are evolved in time. These include dilution upon vapor expansion and resupply of particles from vaporization according to the Clausius-Clapeyron equation at the normal evaporation temperature and various density and temperature acceleration and deceleration mechanisms whose rates are taken from Zel'dovich and Raizer [10].
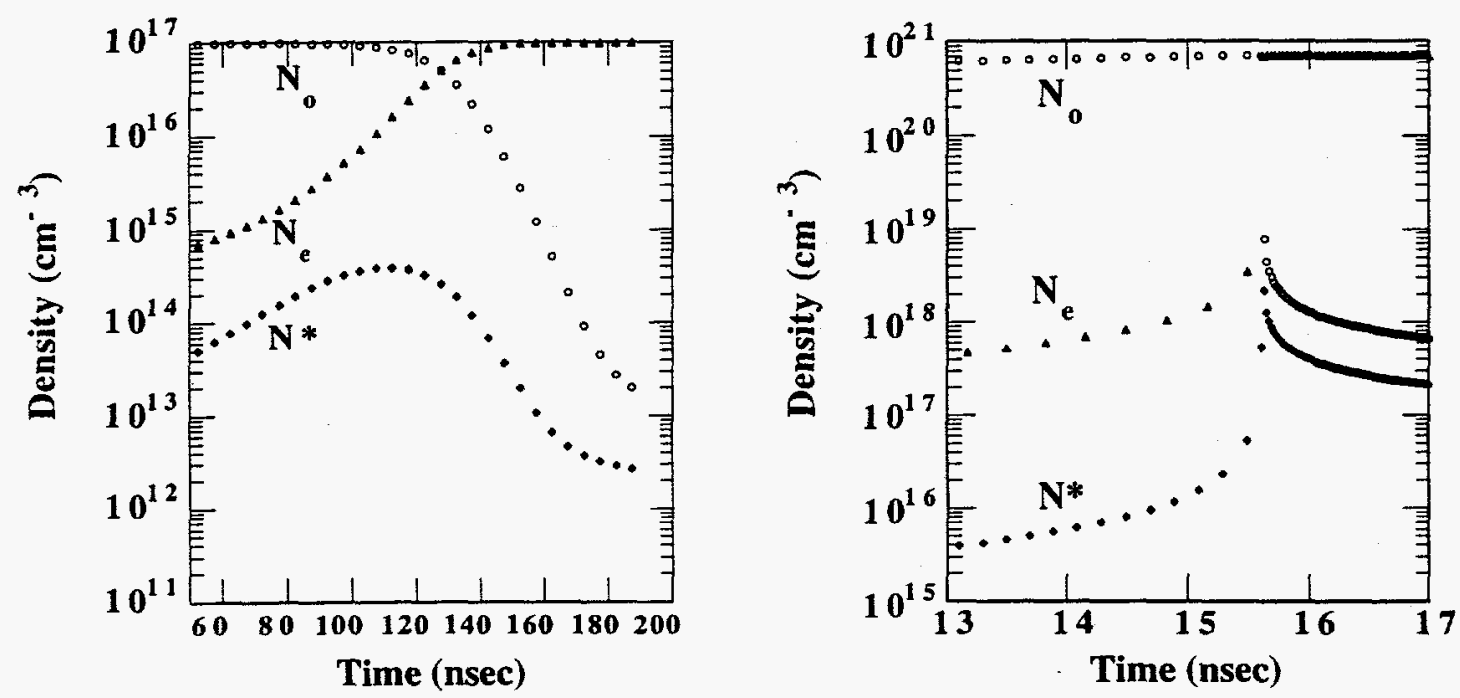

Fig. 2 Results from kinetic model of breakdown for silicon: ground state neutral $\left(\mathrm{N}_{\mathrm{o}}\right)$, excited state neutral $\left(N^{*}\right)$, and electron $\left(N_{e}\right)$ densities in the plume as a function of time for laser energy densities of $5 \mathrm{~J} / \mathrm{cm}^{2}$ (left) and $30 \mathrm{~J} / \mathrm{cm}^{2}$ (right).

The key ingredient of the model is the two-stage process of electron impact excitation from the ground state to the excited state for neutrals at an energy of $5 \mathrm{eV}$ and the subsequent electron 
impact ionization and photoionization of the excited state neutral to a singly charged silicon ion, which requires an additional $3.1 \mathrm{eV}$. This is to be contrasted with the jump from ground state neutral to singly charged ion at an energy of $8.1 \mathrm{eV}$, which is therefore more difficult to achieve with energies of $5 \mathrm{eV}$ per photon in the $\mathrm{KrF}$ laser pulse. Mechanisms for absorption of laser light now include photoionization of the excited state [10], in addition to electron-neutral and electronion inverse Bremstrahlung [6]. Results from the solution of these zero-dimensional rate equations are shown in Fig. 2 where the evolution in time of the densities of electrons, ground state neutrals, and excited state neutrals is displayed for a laser energy density of $5 \mathrm{~J} / \mathrm{cm}^{2}$ on the left and $30 \mathrm{~J} / \mathrm{cm}^{2}$ on the right. Acceleration of the electron density is clear in both cases from an initial state given by the Saha equation for the priming electrons and a Boltzmann distribution for the excited state silicon neutrals. Production of a significant number of electrons and therefore plume breakdown do occur within the laser pulse at the higher energy density of $30 \mathrm{~J} / \mathrm{cm}^{2}$. However, breakdown does not occur within the laser pulse for the lower energy density of $5 \mathrm{~J} / \mathrm{cm}^{2}$. We will compare our transport modeling results with experimental observations at low fluences for which particulates or clusters are absent. Because ionization is difficult to achieve from a theoretical point of view at these low fluences, we have concentrated on neutral fluids for our models of plume transport.

\section{Plume transport}

Experimental observations have shown marked differences between plume expansion in vacuum and in the presence of a higher pressure background gas. These observations are common to a wide range of ablated materials including silicon, carbon, yttrium, and high-temperature superconducting compounds such as YBCO. Ablation in high-pressure ambient gases results in shock waves and expansion fronts propagating through the background gases. Time-of-flight measurements also show two components in the ion probe signals, an energetic component that propagates at vacuum speed and another that is more or less significantly slowed down depending on the pressure of the background gas [2]. We have applied several hydrodynamic, gas dynamic, and collisional models to study plume expansion in vacuum and in a higher pressure background gas. Results from some of these models will be described starting from more qualitative but higher dimensional ones and proceeding to the more detailed and flexible 1-D ones.

To investigate the gross hydrodynamic features of plume transport in vacuum and in background gas, we have used a $2-\mathrm{D}$ gas dynamic model that solves conservation equations for mass density $(\rho)$, momentum $(\rho \vec{v})$, and energy $\left(\rho e+1 / 2 \rho v^{2}\right)$ :

$$
\begin{aligned}
& \frac{\partial}{\partial \mathrm{t}} \rho=-\vec{\nabla} \cdot(\rho \overrightarrow{\mathrm{v}}), \\
& \frac{\partial}{\partial \mathrm{t}}(\rho \overrightarrow{\mathrm{v}})=-\vec{\nabla} \cdot(\mathrm{P} \overrightarrow{\mathrm{I}}+\rho \overrightarrow{\mathrm{v}} \overrightarrow{\mathrm{v}}), \\
& \frac{\partial}{\partial \mathrm{t}}\left(\rho \mathrm{e}+\frac{1}{2} \rho \mathrm{v}^{2}\right)=-\vec{\nabla} \cdot\left\{\overrightarrow{\mathrm{v}}\left[\left(\rho e+\frac{1}{2} \rho \mathrm{v}^{2}\right)+\mathrm{P}\right]\right\} .
\end{aligned}
$$

They are augmented by the following equation of state for internal energy e and pressure P:

$\mathrm{P}=(\gamma-1) \rho e=\frac{\rho}{M} \mathrm{k}_{\mathrm{B}} \mathrm{T}$ with $\gamma=5 / 3$

This model is numerically implemented using finite differences in space and the Rusanov scheme [11] in time. Results from the 2-D model are displayed in Fig. 3 for plume expansion in vacuum (left) and in background gas (right). Contours of total density ( plume plus background) are shown as time progresses from top to bottom. It is evident that strong shocks are generated as the plume expands in presence of the background gas. The plume does in fact snowplow the background gas, giving rise to the crescent feature at the leading edge, which is clearly seen in Fig. 3. 

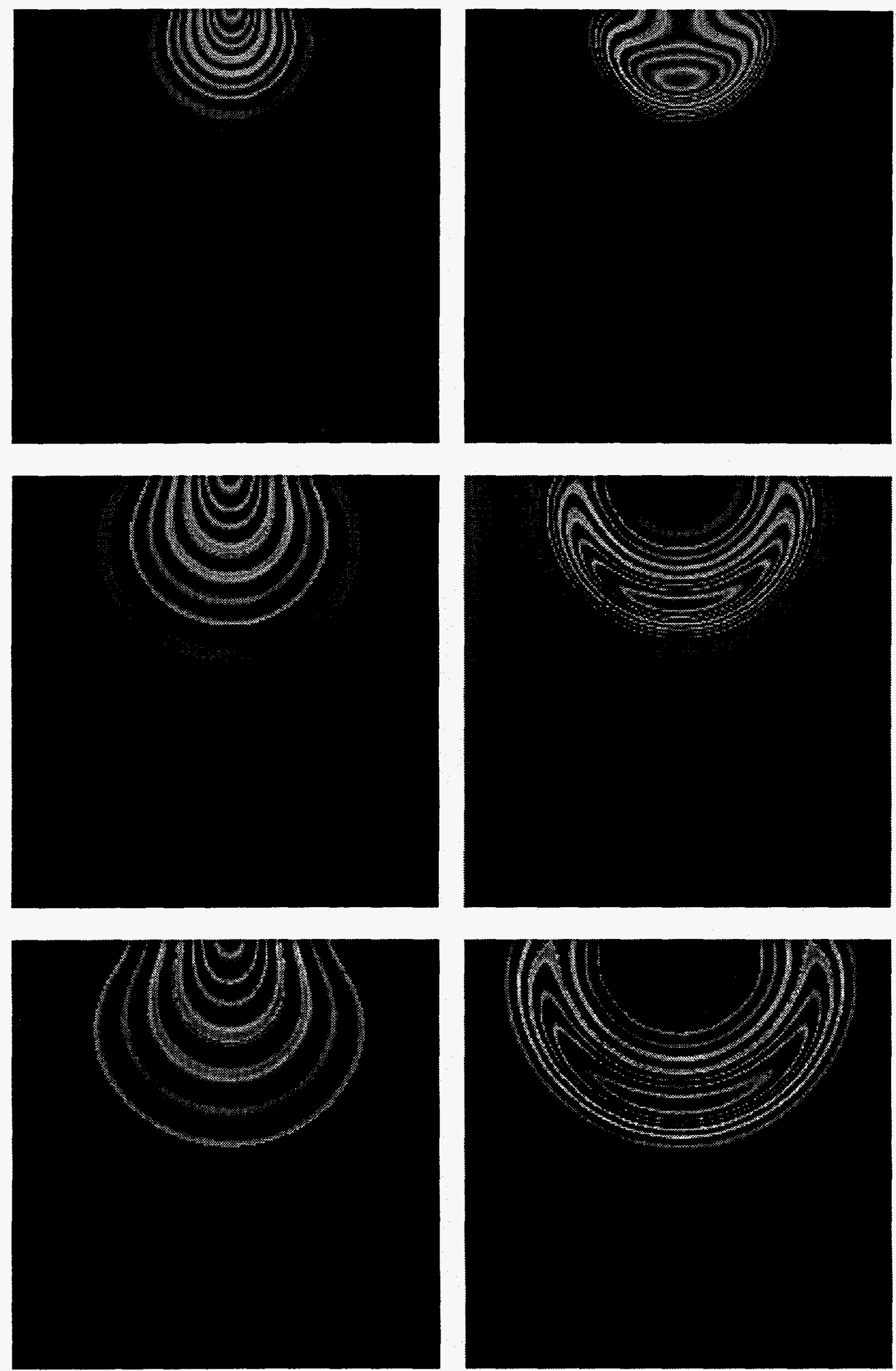

Fig. 3 Results from 2D gas dynamic model: contours of density for plume expansion in vacuum (left) and in background gas (right) as a function of time (top to bottom). 
We note that the gross characteristics of the expansion in vacuum and in background gas are remarkably similar to the light emission patterns experimentally detected using a gated CCD camera [3]. We have also produced similar features [12] using a novel 2-D particle-in-cell hydrodynamic model $[13,14]$ of plume transport in which particles representing elements of the fluid are followed in space and time in the pressure gradient field.

Characteristics of plume expansion in vacuum and in background gas have been examined in more detail using a 1-D gas dynamic model that solves the same conservation equations as the 2-D model, but includes ionization through the Saha equation, and energy input through laser light absorption. The set of equations used in the 1-D model are as follows:

$$
\begin{aligned}
& \frac{\partial}{\partial t} \rho=-\frac{\partial}{\partial x}\left(\rho v_{x}\right)+S_{\rho}, \\
& \frac{\partial}{\partial t}\left(\rho v_{x}\right)=-\frac{\partial}{\partial x}\left(\rho v_{x}^{2}+P-\mu \frac{\partial v_{x}}{\partial x}\right), \\
& \frac{\partial}{\partial t}\left(\rho e+\frac{1}{2} \rho v_{x}^{2}\right)=-\frac{\partial}{\partial x}\left\{v_{x}\left[\left(\rho e+\frac{1}{2} \rho v_{x}^{2}\right)+P-\mu \frac{\partial v_{x}}{\partial x}\right]\right\}+\alpha \Phi(t) e^{-\alpha x}+S_{e},
\end{aligned}
$$

with $\mu$ denoting the viscosity and $P=(1+\eta) \rho \frac{k_{B} T}{M}$. The ionization fraction $\eta$ is determined by a simultaneous solution of the Saha equation:

$$
\frac{\eta^{2}}{1-\eta}=\frac{M}{\rho}\left(\frac{2 \pi m k_{B} T}{h^{2}}\right)^{\frac{3}{2}} e^{-\frac{l_{p}}{k_{B} T}},
$$

and the equation of state:

$$
\rho e=\frac{\rho}{M}\left[\frac{3}{2}(1+\eta) k_{B} T+\eta I_{p}\right]
$$

where $I_{p}$ is the ionization potential. This model is similar to the 1-D models of Vertes and coworkers $[15,16]$, except that the Rusanov scheme is again used to solve the equations [11]. Our model also contains source terms for mass density and energy input denoted by $S_{\rho}$ and $S_{e}$, respectively, in the mass density and energy equation. These source terms allow us to start the calculations with a clean slate and input mass and energy into the transport model according to the results from calculations of laser-target interactions using the Laser8 computer program. The mass density source is then given as $S_{\rho}=n_{l i q} M v_{r s}$ and the energy one as $S_{e}=n_{l i q} M k_{B} T_{v} /(\gamma-1)$, with $v_{r s}$ the recession speed and $n_{l i g}$ the liquid density. For the duration of the laser pulse, these terms provide a dynamic source of mass and energy into the system.

Results from 1-D gas dynamic calculations of silicon plume transport in vacuum with the dynamic source effect and of calculations where the density and temperature profiles at the end of the laser pulse are taken as initial conditions and allowed to freely expand are displayed in Fig. 4. These calculations were performed without ionization of the vapor and without absorption of laser light by the vapor plume. The time evolution of the pressure at the solid surface with the source effect and for free expansion are shown on the left, while the plume front position as a function of time in both of these cases is shown on the right. It is clear from Fig. 4 that high pressure at the surface is maintained for a longer time due to continuous ablation for the duration of the laser pulse compared to the free expansion case where the pressure at the surface rapidly drops as $1 / \mathrm{t}$. As a result, the plume expands with a higher velocity than in the free expansion case as also shown in Fig. 4 where the plume front speed is $\sim 1 \times 10^{6} \mathrm{~cm} / \mathrm{sec}$ with the dynamic source effect compared 
to $\sim 5 \times 10^{5} \mathrm{~cm} / \mathrm{s}$ in the case of free expansion. More details on the dynamic source effect for plume transport in vacuum, including analytical expressions for the steady-state density profile and maximum front velocity, can be found in a companion paper [17].
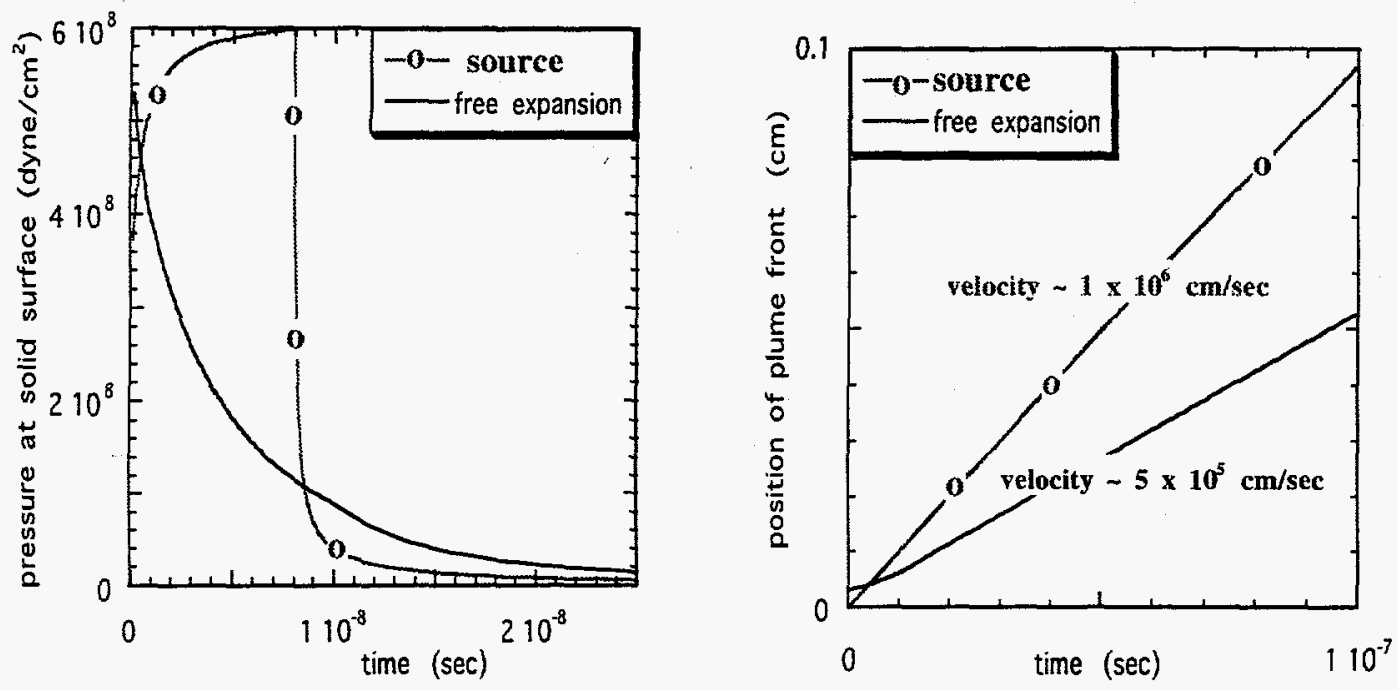

Fig. 4 Results from 1-D gas dynamic model with dynamic source effect from continuous ablation of material within the laser pulse: pressure at the solid surface with dynamic source effect and for free expansion (left) and position of plume front in these same two cases (right) as a function of time.
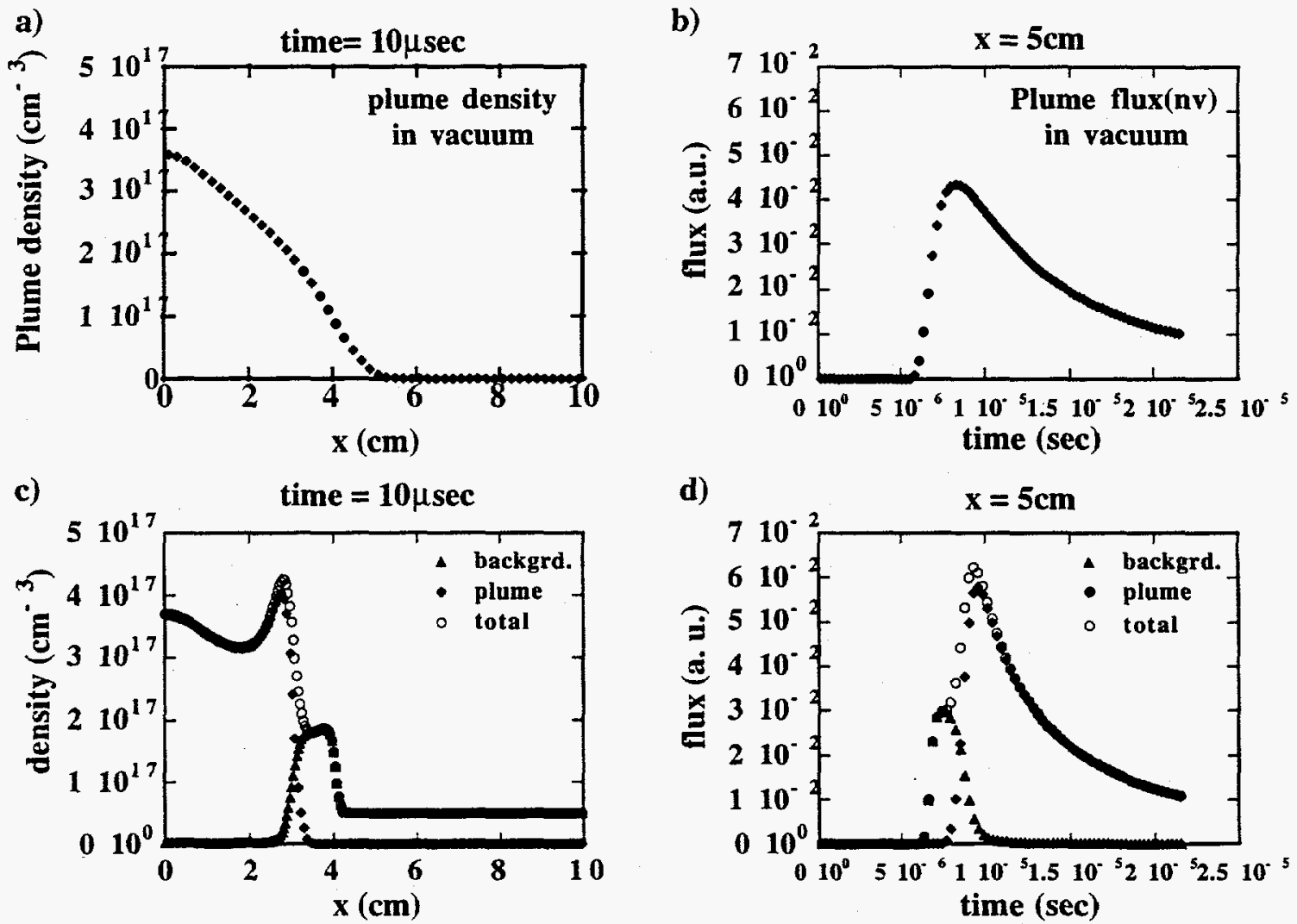

Fig. 5 Results from 1-D gas dynamic calculations of plume free expansion in vacuum and in background gas: a) density profile of plume in vacuum at $10 \mu \mathrm{s}$; b) plume flux in vacuum as a function of time at $5 \mathrm{~cm}$ from the target; c) total, plume, and background density profiles at $10 \mu \mathrm{s}$; d) total, plume, and background fluxes as a function of time at $5 \mathrm{~cm}$ from the target. 
Plume free expansion in vacuum and in a higher pressure background gas has also been studied with our 1-D gas dynamic model, albeit for silicon expanding into a silicon background. For the calculations with background gas presented in Fig. 5, the background density $n_{b}$ was set at $\mathrm{n}_{\mathrm{b}} / \mathrm{n}_{\mathrm{p}}=5 \times 10^{-3}$ compared to the plume density $\mathrm{n}_{\mathrm{p}}$. Comparison of the density profiles in vacuum and in background gas displayed in Fig. 5 shows that the plume snowplows the background gas, which is pushed ahead of the plume, while the expansion of the plume is slowed down in presence of the background gas. The particle fluxes displayed in Fig. 5(d) show that the snowplowed background gas reaches the probe at $5 \mathrm{~cm}$ from the target at plume vacuum speed, with the plume arriving later at the detector because of slowing down from interaction with the background gas.

The results of pursuing the time evolution further are shown in Fig. 6 for a calculation with dynamic source effect and a silicon background gas pressure of $200 \mathrm{mT}$ Trr. The density profile is displayed as a function of distance from the target at four different times in the calculations up to $500 \mu \mathrm{s}$. Snowplowing of the background gas at the leading edge (a); rarefaction of the plume (b); slowdown and turnaround of the plume peak, the peak between target and front, by the snowplowed and piled-up background gas at the leading edge (c); and the subsequent reflection of the plume peak from the target (d) lead to multiple shocks between target and front, as observed in experiments performed in high background gas pressure [2].
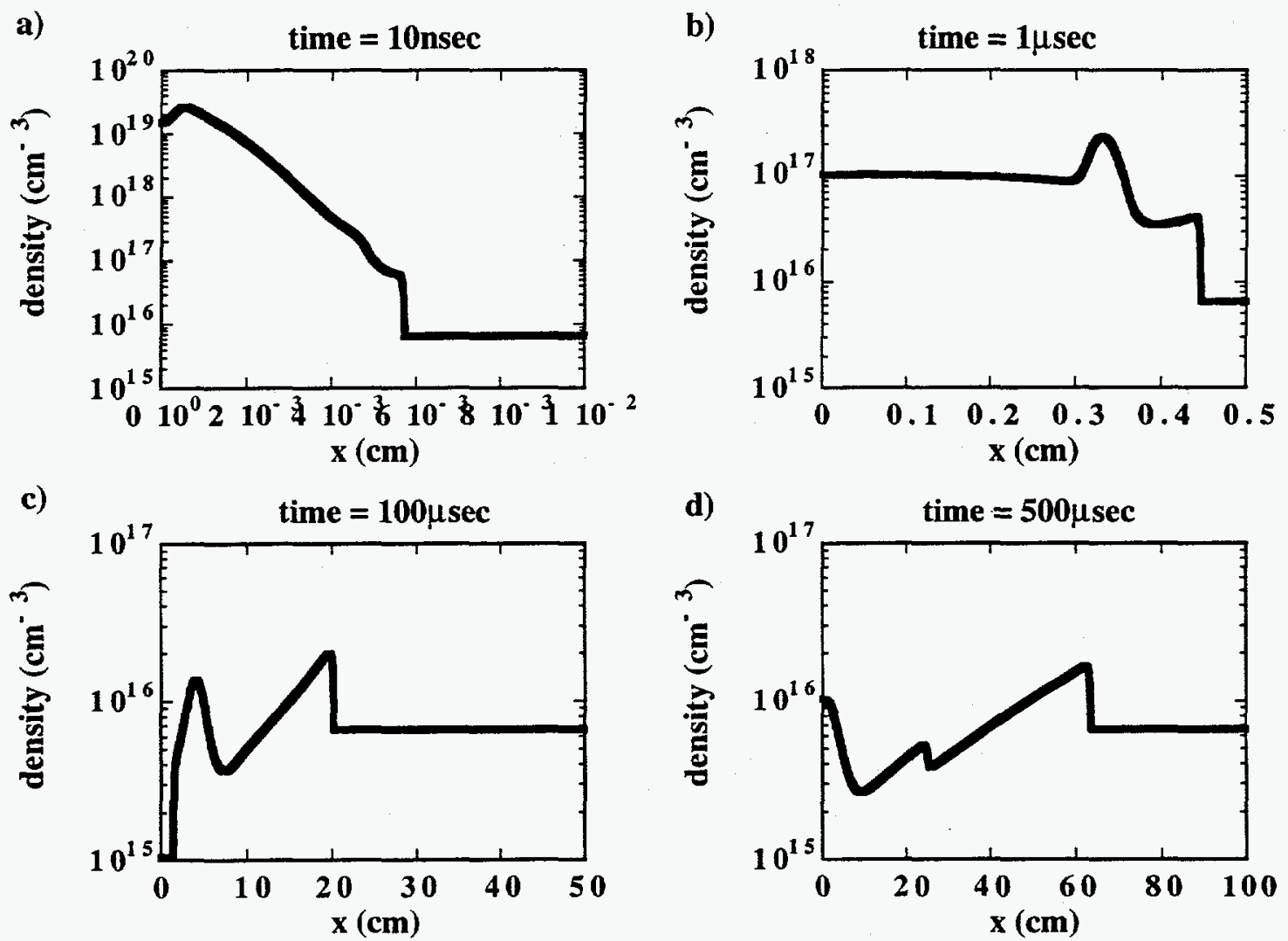

Fig. 6 Results from 1-D gas dynamic calculations of plume expansion in background gas at a pressure of 200 mTorr: density profile as a function of distance from the target at various times during the evolution.

Other models of plume-background interactions are also being considered in which the background and the plume are treated as two distinct species, but which include collisional interactions between them. One such model [18] treats each species using a set of fluid equations for density and momentum:

$$
\frac{\partial}{\partial t} n_{s}+\frac{\partial}{\partial x}\left(n_{s} v_{s}\right)=0
$$




$$
\frac{\partial}{\partial t}\left(n_{s} v_{s}\right)+\frac{\partial}{\partial x}\left[\frac{P_{s}}{m_{s}}+v_{s}\left(n_{s} v_{s}\right)\right]=-\frac{n_{s}}{m_{s}} \sum_{s^{\prime}} \frac{m_{s} m_{s^{\prime}}}{m_{s}+m_{s^{\prime}}} C_{s s^{\prime}} n_{s^{\prime}}\left(v_{s}-v_{s^{\prime}}\right),
$$

with a collisional drag term between species s (plume) and species s' (background) included on the right-hand side of the momentum evolution equations. The strength of the interaction is denoted by $\mathrm{C}_{\mathrm{ss}}{ }^{\prime}$ which is taken to be constant for the calculations presented here. Note that the drag term is momentum conserving when summed over the two species. The pressure is taken to be $\mathrm{P}_{\mathrm{S}}=\mathrm{n}_{\mathrm{s}} \mathrm{k}_{\mathrm{B}} \mathrm{T}_{\mathrm{s}}$. Results from a calculation with this two-species (silicon in silicon) model are displayed in Fig. 7 where total, plume and background density profiles at a particular time and fluxes at $5 \mathrm{~cm}$ from the target as a function of time are shown. It is clear from the density profiles that the plume has penetrated through the background gas and that the background gas is being dragged along by the plume. The fluxes show that the plume component gets to the probe first at essentially vacuum speed, with the dragged background gas reaching the detector some time later.
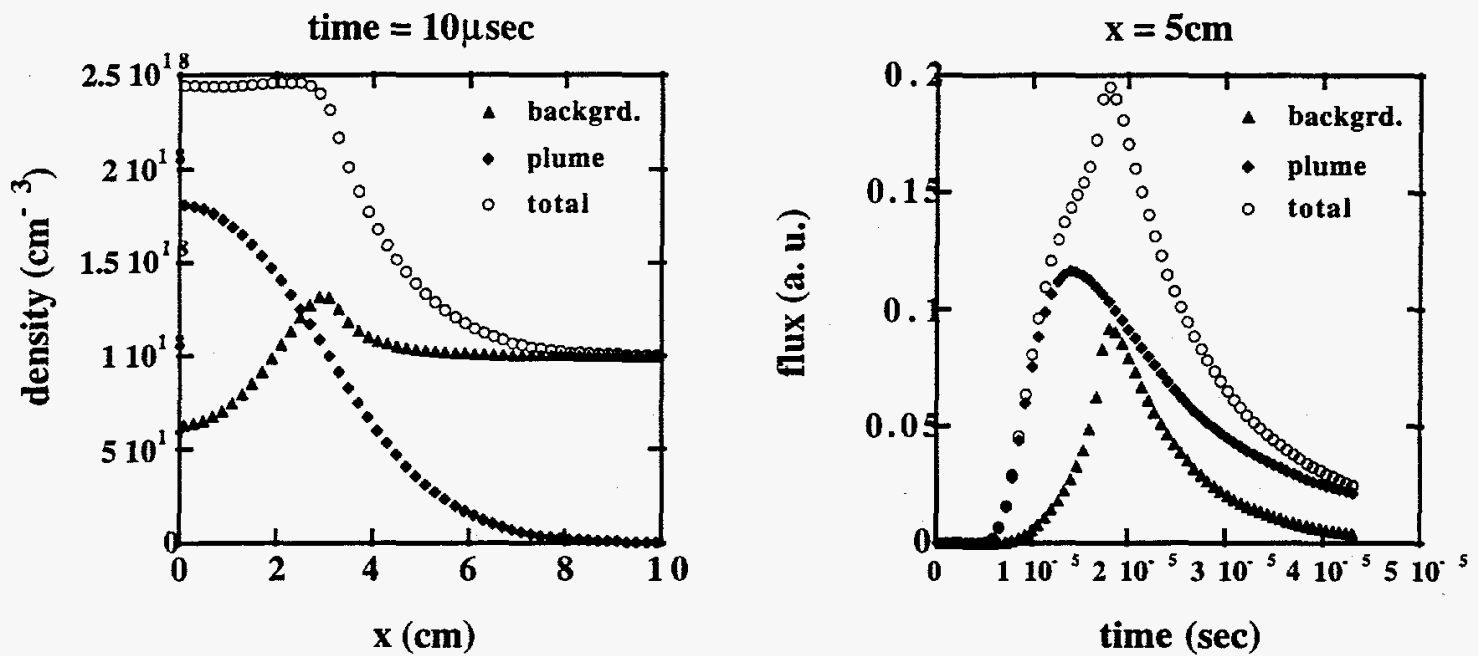

Fig. 7 Results from hydrodynamic model with two interpenetrating species: background, plume, and total densities (left) and fluxes (right).

Other approaches that show promise in elucidating the phenomena that take place when the plume interacts with a background gas include a model first proposed by Koopman and Goforth [19] and a scattering model we have recently developed. The model of Koopman and Goforth relies on a fully ionized layer of background gas to scatter the plume ions through ion-ion collisions. It is only by including this interaction that two velocity components are observed in the plume fluxes detected $5 \mathrm{~cm}$ from the target in the numerical calculations. Our scattering model includes plume-plume, plume-background, and background-background collisional interactions with cross sections that depend on the difference of the velocities to various inverse powers. The calculated fluxes at a certain distance from the target indicate that background or plume get to the probe first depending on whether the background gas is lighter, such as helium, or heavier, such as argon, than the silicon plume, with velocities exceeding plume vacuum speed in the helium case under conditions appropriate for elastic collisions.

\section{PLUME-SUBSTRATE INTERACTIONS}

Time-of-flight measurements [2], as well as the model calculations presented here, clearly show that material reaches the deposition substrate at speeds equal to and even exceeding plume speed in vacuum even in the presence of a background gas, followed by a slower moving component whose velocity depends on background pressure. It is expected that the fast-traveling component may cause film damage due to the high kinetic energy of these particles. In the case of deposition of copper films, time-of-flight measurements indicate that the fast-moving particles can have kinetic 
energies in excess of $200 \mathrm{eV}$, while the energies of the slow-moving particles are $<100 \mathrm{eV}$ under normal ablation conditions.

We have performed atomistic calculations using the embedded atom method of molecular dynamics to assess the effect of particle kinetic energies on the film quality by pulsed laser deposition from a copper target. A copper particle above the $\mathrm{Cu}(100)$ surface is allowed to hit the surface at velocities corresponding to kinetic energies ranging from $100 \mathrm{eV}$ to $200 \mathrm{eV}$. The most probable point on the surface for the incoming particle to hit was found to be the open crystal channel along the $\langle 100\rangle$ direction on $\mathrm{Cu}(100)$. Trajectories of the particles and substrate atoms are recorded as a function of time. As seen in Fig. 8, the critical energy for penetration of a copper particle into the $\mathrm{Cu}(100)$ surface is found to be $\sim 150 \mathrm{eV}$. At this energy, the particle almost touches the surface atoms but does not penetrate. Figure 8 also shows that an energetic copper particle with a kinetic energy of $200 \mathrm{eV}$ can however penetrate the first layer of the $\mathrm{Cu}(100)$ surface. The hopping distances for this $200 \mathrm{eV}$ particle are large initially and gradually decrease as the particle loses its kinetic energy. It finally comes to rest after hopping about $5 \mathrm{~A}$ along the $<110\rangle$ surface channel. It is therefore possible for $200 \mathrm{eV}$ particles to cause permanent mechanical damage according to these molecular dynamics calculations.

\section{Critical Kinetic Energy for Penetration: $150 \mathrm{eV}$ for Neutrals Bombardment of $\mathrm{Cu}(100)$}

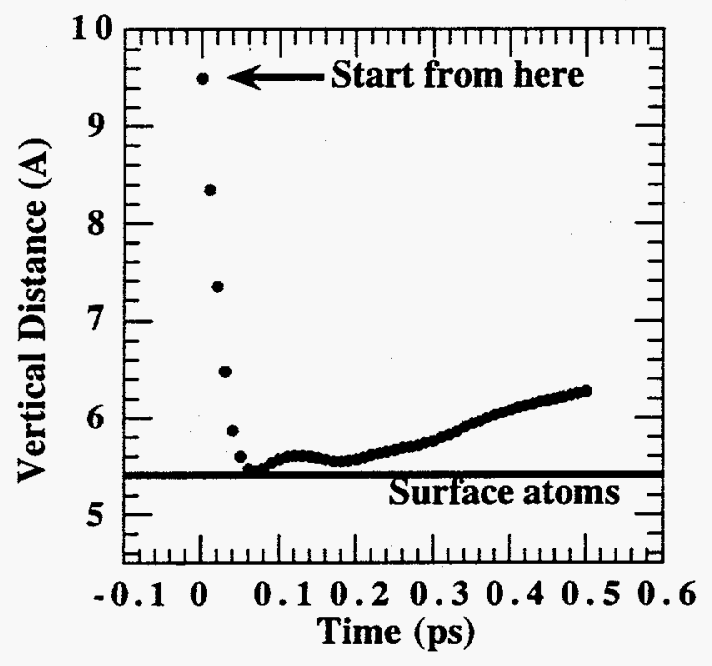

$200 \mathrm{eV}$ Neutrals Bombardment of $\mathrm{Cu}(100)$

Trajectory of the Energetic Particle

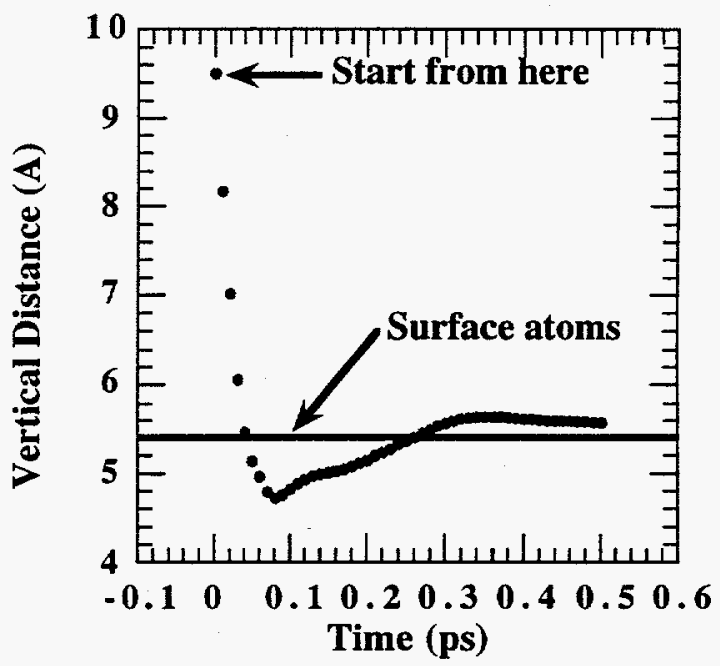

Fig. 8 Results from molecular dynamics calculations using the embedded atom method of film damage by energetic particles: vertical trajectories of a copper plume particle with kinetic energies of $150 \mathrm{eV}$ (left) and $200 \mathrm{eV}$ (right).

\section{SUMMARY}

We have described a global approach to modeling of the laser ablation process with several physics and computational models applied to laser-target interactions, plume transport in vacuum and in background gas, and plume-substrate interactions. The remaining challenges include extending models so far applied to silicon to more complex materials, that is, progress from elemental materials to compounds. They also entail continued quantitative comparisons with experiments, as well as model integration. 


\section{ACKNOWLEDGMENTS}

This research is sponsored at the Oak Ridge National Laboratory (ORNL) by Laboratory Directed Research and Development (LDRD) Funds and by the Division of Materials Sciences, U. S. Department of Energy, under contract DE-AC05-84OR21400 with Martin Marietta Energy Systems, Inc. K. R. Chen and Chun-Li Liu are supported by an appointment to the ORNL Research Associate Program administered jointly by the Oak Ridge Institute for Science and Education and ORNL. Alex Puretzky is on assignment to the ORNL from the Institute of Spectroscopy, Russian Academy of Sciences, Troitsk, Russia.

\section{REFERENCES}

1. J. T. Cheung, Pulser Laser Deposition of Thin Films, ed. D. B. Chrisey and G. K. Hubler (Wiley, New York ,1994) pp. 1-22.

2. D. B. Geohegan, A. A. Puretzky, J. N. Leboeuf, K. R. Chen, C. L. Liu, R. F. Wood, J. M. Donato, and D. Lowndes, Paper Q1.5, these proceedings.

3. D. B. Geohegan, Appl. Phys. Lett. 60, 2732 (1992).

4. R. F. Wood and G. A. Geist, Phys. Rev. Lett. 57, 873 (1986).

5. R. F. Wood and G. A. Geist, Phys. Rev. B34, 2606 (1986).

6. A. Anders, A Formulary for Plasma Physics (Akademie-Verlag, Berlin, 1990), pp. 159-187.

7. T. W. Johnston, and J. M. Dawson, Phys. Fluids 16, 722 (1973).

8. C. L. Liu, J. N. Leboeuf, R. F. Wood, D. B. Geohegan, J. M. Donato, K. R. Chen, and A. A. Puretzky, Paper Q13.4, these proceedings.

9. D. I. Rosen, J. Mitteldorf, G. Kothandaraman, A. N. Pirri, and E. R. Pugh, J. Appl. Phys. 53, 3190 (1982).

10. Ya. B. Zel'dovich, and Yu. P. Raizer, Physics of Shock Waves and High-Temperature Hydrodynamic Phenomena (Academic Press, New York and London, 1966).

11. G. A. Sod, Journ. Comput. Phys 27, 1 (1978).

12. C. L. Liu, J. N. Leboeuf, R. F. Wood, D. B. Geohegan, J. M. Donato, K. R. Chen, and A. A. Puretzky, Beam-Solid Interactions for Materials Synthesis and Characterization. ed. D. E. Luzzi, T. F. Heinz, M. Iwaki, D. C. Jacobson (Mater. Res. Soc. Proc. 351, Boston, Mass., 1994).

13. J. N. Leboeuf, T. Tajima, and J. M. Dawson, Journ. Comput. Phys 31, 379 (1979).

14. F. Brunel, J. N. Leboeuf, T. Tajima, J. M. Dawson, M. Makino, and T. Kamimura, Journ. Comput. Phys. 43, 268 (1981).

15. A. Vertes, P. Juhasz, M. D. Wolf, and R. Gijbels, Scanning Microscopy 2, 1853 (1988).

16. L. Balazs, R. Gijbels, and A. Vertes, Analytical Chemistry 63, 314 (1991).

17. K. R. Chen, J. N. Leboeuf, D. B. Geohegan, J. M. Donato, R. F. Wood, and C. L. Liu, Paper Q2.1, these proceedings.

18. P. W. Rambo, and J. Denavit, Journ. Comput. Phys. 98, 317 (1992).

19. D. W. Koopman, and R. R. Goforth, Phys. Fluids 17, 1560 (1974).

\section{DISCLAIMER}

\footnotetext{
This report was prepared as an account of work sponsored by an agency of the United States Government. Neither the United States Government nor any agency thereof, nor any of their bility for the accuracy, completeness, or or implied, or assumes any legal liability or responsiprocess disclosed, or represents that its usefulness of any information, apparatus, product, or ence herein to any specific commercial use would not infringe privately owned rights. Refermanufacturer, or otherwise does not product, process, or service by trade name, trademark, mendation, or favoring by the United States Gonstitute or imply its endorsement, recomand opinions of authors expressed herein dovernment or any agency thereof. The views United States Government or any agency thereof. nocessarily state or reflect those of the
} 